\title{
SOME REMARKS ON REAL-VALUED MEASURABLE CARDINALS
}

\author{
ANDRZEJ SZYMAŃSKI
}

(Communicated by Thomas J. Jech)

\begin{abstract}
We consider the set $[\omega]^{\omega}$ and the cofinality of the set ${ }^{\kappa} \lambda$ assuming that some cardinals are endowed in total measures.
\end{abstract}

Introduction. A cardinal $\kappa$ is real-valued measurable if there exists a $\kappa$ complete atomless probability measure on $P(\kappa)$, the set of all subsets of the cardinal $\kappa$. The status and the philosophy concerned e.g. real-valued measurable cardinals have been detailed and presented in a survey paper by A. Kanamori and M. Magidor $[\mathbf{K M}]$. We shall concentrate mainly on two problems: the cofinality of sets of functions with respect to eventual domination and some combinatorics on $\omega$, both assuming the existence of some total measures.

T. Jech and K. Prikry [JP] showed, assuming $2^{\omega}$ is real-valued measurable, that the cofinality of the set of all functions from $\omega_{1}$ into $\omega$ equals $2^{\omega}$. We extend and complete their result. We show (Theorems 1 and $3, \S 2$ ), under the same assumption about $2^{\omega}$, that if $\kappa<2^{\omega}$ is regular and $\omega \leq \lambda \leq \kappa$, then the cofinality of the set of all functions from $\kappa$ into $\lambda$ equals $2^{\omega}$ provided $\operatorname{cf}(\lambda)=\omega$ and the cofinality is less than $2^{\omega}$ provided $\operatorname{cf}(\lambda)>\omega$.

It is well known that under Martin's axiom, $2^{\omega}$ cannot be real-valued measurable. The reason is, that under Martin's axiom the cofinality of the set of all functions from $\omega$ into $\omega$ equals $2^{\omega}$ while assuming $2^{\omega}$ is real-valued measurable, the cofinality is $<2^{\omega}$ (see also Theorem 4, §2). We give some other reasons for which some cardinals cannot carry total measures. We show (Corollary 2, §1) that if there exists a maximal $\kappa$-tower on $\omega$, then $\kappa$ is not real-valued measurable cardinal. It has been shown by $\mathrm{S}$. Hechler $[\mathbf{H}]$ that each regular cardinal $\kappa, \omega<\kappa \leq 2^{\omega}$ can be (consistently) the length of some maximal $\kappa$-tower on $\omega$.

Throughout the paper we use standard set-theoretical notation. For example $[\omega]^{\omega}$ is used to denote the set of all infinite subsets of the least infinite ordinal $\omega$. All undefined terms can be found in $[\mathbf{J}]$.

1. Let ${ }^{\omega} \omega$ denote the set of all functions from $\omega$ into $\omega$. For two arbitrary functions $f, g \in{ }^{\omega} \omega$ we set $f<_{*} g$ iff $f(n)<g(n)$ for all but finitely many $n \in \omega$.

Received by the editors July 30, 1986 and, in revised form, November 20, 1987. Presented at Spring Topology Conference, Lafayette, April 3-5, 1986.

1980 Mathematics Subject Classification (1985 Revision). Primary 03E55, 04A20.

Key words and phrases. Probability measure, cofinality, tower.

The author wishes to express his gratitude to the Department of Mathematics and Computer Sciences of the Youngstown State University for its support and hospitality during the preparation of this work. 
LEMMA 1. Suppose $\mathscr{F} \subset{ }^{\omega} \omega$ and $\mu$ is a $\sigma$-additive probability measure on $P(\mathscr{F})$. Then there exists a $g \in{ }^{\omega} \omega$ such that the set $\left\{f \in \mathscr{F}: f<_{*} g\right\}$ is of $\mu$-measure 1 .

PROOF. For each $m \in \omega$ we shall define a function $g_{m} \in{ }^{\omega} \omega$ such that the set $F_{m}=\left\{f \in \mathscr{F}: f(n) \leq g_{m}(n)\right.$ for all $\left.n \in \omega\right\}$ is of $\mu$-measure at least $1-1 / m$. To do this, let $l \in \omega$ be such that $\sum_{k=l}^{\infty}\left(1 / 2^{k}\right)<1 / m$. For every $n \in \omega$ choose $k_{n} \in \omega$ such that the set $A_{n}=\left\{f \in \mathscr{F}: f(n) \leq k_{n}\right\}$ is of $\mu$-measure at least $1-1 / 2^{l+n}$ (this is possible because for every $\left.n \in \omega, \lim _{m \rightarrow \infty} \mu(\{f \in \mathscr{F}: f(n) \leq m\})=1\right)$. If we put $g_{m}(n)=k_{n}$ for every $n \in \omega$, then the set $F_{m}$ contains the set $\bigcap\left\{A_{n}: n \in \omega\right\}$ which is of $\mu$-measure at least $1-\sum_{k=l}^{\infty}\left(1 / 2^{k}\right)$ which is greater than $1-1 / m$. Taking arbitrary function $g \in{ }^{\omega} \omega$ such that $g_{m}<_{*} g$ we see that the set $\left\{f \in \mathscr{F}: f<_{*} g\right\}$ is of $\mu$-measure 1 because it contains each of the sets $F_{m}$.

COROLLARY 1. Suppose that there exists a family $\left\{f_{\alpha}: \alpha<\kappa\right\} \subset{ }^{\omega} \omega$ such that $\alpha<\beta<\kappa$ implies $f_{\alpha} \leq_{*} f_{\beta}$ and for every $f \in \omega_{\omega} \omega$ there exists an $\alpha, \alpha<\kappa$, such that $\left|\left\{n: f(n) \leq f_{\alpha}(n)\right\}\right|=\omega$. Then $\kappa$ is not a real-valued measurable cardinal.

LEMMA 2. Suppose $\mathscr{S} \subset[\omega]^{\omega}$ and $\mu$ is a $\sigma$-additive probability measure on $P(\mathscr{S})$. Then there exists an $x \in[\omega]^{\omega}$ such that the set $\{s \in \mathscr{S}:|s \cap x|=\omega=$ $|s \cap(\omega-x)|\}$ is of $\mu$-measure 1 .

PROOF. For $s \in \mathscr{S}$ let $f_{s} \in{ }^{\omega} \omega$ be such that $f_{s}(n)=(n+1)$ th element of the set $s$. By Lemma 1, there exists a strongly increasing function $g \in{ }^{\omega} \omega$ such that the set $A=\left\{s \in \mathscr{S}: f_{s}<_{*} g\right\}$ is of $\mu$-measure 1. Let $h(0)=1$ and $h(n+1)=g(h(n)-1)$, for $n \in \omega$. Put $x=\bigcup\{[h(2 n), h(2 n+1)): n \in \omega\}$. It is easy to verify that for every $s \in A,|s \cap x|=\omega=|s \cap(\omega-x)|$ (see [vD]).

For $\xi$, an ultrafilter on $\omega$, put $\chi(\xi)=\inf \{|B|: B \subset \xi$ and for every $x \in \xi$ there exists $b \in B$ such that $b \subset x\}$-the character of $\xi$.

LEMMA 3. Let $\xi$ be a free ultrafilter on $\omega$ and let $\kappa=\chi(\xi)$. Suppose that there exists a $\sigma$-additive probability measure $\mu$ on $P(\kappa)$ such that each set of $\mu$-measure 1 has size $\kappa$. Then there exists an $A \subset \xi$ such that $|A|<\kappa$ and for every $x \in[\omega]^{\omega}$ ther exists an $a \in A$ satisfying $|x-a|=\omega$.

PROOF. Let $\left\{a_{\alpha}: \alpha<\kappa\right\} \subset \xi$ be such that every $x \in \xi$ contains some $a_{\alpha}$. We shall show that for some $\beta<\kappa$ the set $\left\{a_{\alpha}: \alpha<\beta\right\}$ satisfies our theorem. If no, then for every $\beta<\kappa$ there would exist $s_{\beta} \in[\omega]^{\omega}$ such that $\left|s_{\beta}-a_{\alpha}\right|<\omega$ for every $\alpha<\beta$. By Lemma 2, there exists an $x \in[\omega]^{\omega}$ such that the set $C=\{\alpha<$ $\left.\kappa:\left|s_{\alpha} \cap x\right|=\omega=\left|s_{\alpha} \cap(\omega-x)\right|\right\}$ would be of $\mu$-measure 1 . This set has the size $\kappa$. Suppose $x$ belongs to $\xi$ (in the case $\omega-x \in \xi$ we proceed in the same manner). Then there would exist an $\alpha, \alpha<\kappa$, such that $a_{\alpha} \subset x$. Choose $\gamma, \gamma \in C$, greater than $\alpha$. Then $\left|s_{\gamma}-a_{\alpha}\right|<\omega$ and $\left|s_{\gamma} \cap(\omega-x)\right|=\omega$, which is impossible.

Following S. Hechler $[\mathbf{H}]$ we say that a family $\left\{T_{\alpha}: \alpha<\kappa\right\} \subset[\omega]^{\omega}$ is a $\kappa$-tower if $\alpha<\beta<\kappa$ implies $\left|T_{\beta}-T_{\alpha}\right|<\omega$.

LEMMA 4. Let $\kappa$ be a regular cardinal and suppose that there exists a $\sigma$-additive probability measure $\mu$ on $P(\kappa)$ such that each set of $\mu$-measure 1 has size $\kappa$. If $T=\left\{T_{\alpha}: \alpha<\kappa\right\}$ is a $\kappa$-tower, then there exists a family $\left\{D_{n}: n \in \omega\right\}$ of pairwise disjoint subsets of $\omega$ such that $\left|T_{\alpha} \cap D_{n}\right|=\omega$ for every $\alpha<\kappa$ and $n<\omega$. 
ProOF. Let $\xi$ be a free ultrafilter containing $T$. There exists a $D_{0} \in \xi$ such that $\left|T_{\alpha}-D_{0}\right|=\omega$ for every $\alpha<\kappa$ (otherwise, in virtue of regularity of $\kappa$, we would have $\kappa=\chi(\xi)$. But then, in virtue of Lemma 3, there would exist $A \subset \xi$ such that $|A|<\kappa$ and for every $\alpha<\kappa$ there exists an $a \in A$ satisfying $\left|T_{\alpha}-a\right|=\omega$. Since $|A|<\kappa$ and $\kappa$ is regular there would exist an $a_{0} \in A$ such that $\left|T_{\alpha}-\alpha_{0}\right|=\omega$ for every $\alpha<\kappa$, which is impossible). Suppose we have defined pairwise disjoint sets $D_{0}, \ldots, D_{n}$ such that $\left|D_{j} \cap T_{\alpha}\right|=\omega$ for every $\alpha<\kappa$ and $j \leq n$ and $\mid T_{\alpha}-$ $\left(D_{0} \cup \cdots \cup D_{n}\right) \mid=\omega$ for every $\alpha<\kappa$. Let $\eta$ be a free ultrafilter containing the $\kappa$-tower $\tilde{T}=\left\{T_{\alpha}-\left(D_{0} \cup \cdots \cup D_{n}\right): \alpha<\kappa\right\}$. There exists a $D_{n+1} \in \eta$ such that $D_{n+1} \subset \omega-\left(D_{0} \cup \cdots \cup D_{n}\right)$ and $\left|T_{\alpha}-\left(D_{0} \cup \cdots \cup D_{n} \cup D_{n+1}\right)\right|=\omega$ for every $\alpha<\kappa$. The constructed sequence $\left\{D_{n}: n<\omega\right\}$ has the required properties.

LEMMA 5. Let $\mu$ be a $\sigma$-additive probability measure on a $\sigma$-field $M$ of subsets of a set $X$. If $\varepsilon$ is a positive number and $\left\{Z_{n}: n<\omega\right\} \subset M$ is such that $\mu\left(Z_{n}\right) \geq \varepsilon$ for each $n<\varepsilon$ then there exists a set $A \in M$ such that $\mu(A) \geq \varepsilon$ and $\mid\{n<\omega: a \in$ $\left.Z_{n}\right\} \mid=\omega$ for every $a \in A$.

PROOF. Let $A_{n}=\bigcup\left\{Z_{m}: m \geq n\right\}$ and $A=\bigcap\left\{A_{n}: n<\omega\right\}$.

THEOREM 1. Suppose that $\kappa$ is a regular cardinal and that there exists a $\sigma$ additive probability measure $\mu$ on $P(\kappa)$ such that each set of positive $\mu$-measure has size $\kappa$. If $T=\left\{T_{\alpha}: \alpha<\kappa\right\}$ is a $\kappa$-tower, then there exists an infinite set $Z$ such that $\left|Z-T_{\alpha}\right|<\omega$ for every $\alpha<\kappa$.

PROOF. In virtue of Lemma 4, there exists a family $\left\{D_{n}: n<\omega\right\}$ of pairwise disjoint sets such that $\left|T_{\alpha} \cap D_{n}\right|=\omega$ for every $\alpha<\kappa$ and $n<\omega$. Let $d_{n m}$ be the $(m+1)$ th element of $D_{n}$ in the natural order. For every $\alpha<\kappa$ define a function $f_{\alpha} \in{ }^{\omega} \omega$ by the rule: $f_{\alpha}(n)=\inf \left\{m: d_{n m} \in T_{\alpha} \cap D_{n}\right\}$. Notice that $f_{\alpha} \leq_{*} f_{\beta}$ whenever $\alpha \leq \beta<\kappa$. In virtue of Lemma 1 , there exists a function $g \in{ }^{\omega} \omega$ such that the set $\left\{\alpha<\kappa: f_{\alpha}<_{*} g\right\}$ is of $\mu$-measure 1. Hence $f_{\alpha}<_{*} g$ for every $\alpha<\kappa$. Put $E_{n}=\left\{d_{n m}: m \leq g(n)\right\}$. Notice that $E_{n}$ is a finite subset of $D_{n}$ for every $n<\omega$ and, if $f_{\alpha}(n) \leq g(n)$, then $T_{\alpha} \cap E_{n} \neq \varnothing$. Hence $\left|\left\{n<\omega: T_{\alpha} \cap E_{n}=\varnothing\right\}\right|<\omega$ for every $\alpha<\kappa$.

For a positive number $\varepsilon$ put $Z_{\varepsilon}=\left\{n<\omega: \mu\left(\left\{\alpha<\kappa: n \in T_{\alpha}\right\}\right) \geq \varepsilon\right\}$. Then $\left|Z_{\varepsilon}-T_{\alpha}\right|<\omega$ for every $\alpha<\kappa$. Indeed, if $\left|Z_{\varepsilon}-T_{\gamma}\right|=\omega$ for some $\varepsilon>0$ and $\gamma<\kappa$, then, in virtue of Lemma 5 , there will exist a set $A \subset \kappa$ such that $\mu(A) \geq \varepsilon$ and $\left|\left\{n \in Z_{\varepsilon}-T_{\gamma}: n \in T_{\alpha}\right\}\right|=\omega$ for every $\alpha \in A$ (here, we apply Lemma 5 to the infinite family $\left\{Z_{n}: n \in Z_{\varepsilon}-T_{\gamma}\right\}$, where $Z_{n}=\left\{\alpha<\kappa: n \in T_{\alpha}\right\}$ ). The set $A$ has the size $\kappa$. Therefore there exists a $\beta \in A$ such that $\gamma<\beta$. But then $\left|\left(Z_{\varepsilon}-T_{\gamma}\right) \cap T_{\beta}\right|=\omega$. In particular, $\left|T_{\beta}-T_{\gamma}\right|=\omega$, which is impossible.

Our theorem will be completely proven when we show that $Z_{\varepsilon}$ is an infinite set for some $\varepsilon>0$.

Suppose to the contrary that it is not the case. Hence the set $Z_{1 / 6}$ is finite. Without loss of generality we may now assume that $\mu\left(\left\{\alpha<\kappa: m \in T_{\alpha}\right\}\right)<\frac{1}{6}$ for every $m \in \bigcup\left\{E_{n}: n<\omega\right\}$. Divide $\omega$ arbitrarily into infinitely many infinite pieces, say $\omega=N_{1} \cup N_{2} \cup \cdots$, and then we put $K_{n}=\bigcup\left\{E_{i}: i \in N_{n}\right\}$. Notice that $K_{n} \cap K_{m}=\varnothing$ whenever $n \neq m$ and that $\left|K_{n} \cap T_{\alpha}\right|=\omega$ for every $\alpha<\kappa$ and $n<\omega$. For every $n<\omega$ we will choose a finite set $s_{n} \subset K_{n}$ such that $\frac{1}{3} \leq$ $\mu\left(\left\{\alpha<\kappa: s_{n} \cap T_{\alpha} \neq \varnothing\right\}\right)<\frac{1}{2}$. To do this, let us fix $j<\omega$ and let us consider the 
sets $c_{n}=\left\{\alpha<\kappa: n \cap K_{j} \cap T_{\alpha} \neq \varnothing\right\}$. They form an increasing sequence of subsets of $\kappa$ such that $\bigcup\left\{c_{n}: n \in \omega\right\}=\kappa$. Hence for some $n, \mu\left(c_{n}\right) \geq \frac{1}{3}$. Let $n_{0}$ be the least such number. Hence $\mu\left(c_{n_{0}-1}\right)<\frac{1}{3}$. However $c_{n_{0}}=c_{n_{0}-1} \cup\left\{\alpha<\kappa: n_{0}-1 \in T_{\alpha}\right\}$. Hence $\mu\left(c_{n_{0}}\right) \leq \mu\left(c_{n_{0}-1}\right)+\mu\left(\left\{\alpha<\kappa: n_{0}-1 \in T_{\alpha}\right\}\right)<\frac{1}{3}+\frac{1}{6}<\frac{1}{2}$. It is enough to put $s_{j}=n_{0} \cap K_{j}$.

To get a contradiction we will apply Lemma 5 twice: first to the family $W_{n}=$ $\left\{\alpha<\kappa: s_{n} \cap T_{\alpha}=\varnothing\right\}, n<\omega$, and then to some subfamily of the family $W_{n}^{\prime}=$ $\left\{\alpha<\kappa: s_{n} \cap T_{\alpha} \neq \varnothing\right\}, n<\omega$. For this, notice that $W_{n}^{\prime}=\kappa-W_{n}, \mu\left(W_{n}^{\prime}\right) \geq \frac{1}{3}$, $\mu\left(W_{n}\right) \geq \frac{1}{2}$ for every $n<\omega$.

By a weak application of Lemma 5 to the family $\left\{W_{n}: n<\omega\right\}$ we get the existence of a $\gamma, \gamma<\kappa$, such that $\Sigma=\left\{n<\omega: \gamma \in W_{n}\right\}$ is an infinite set. Now, by the full application of Lemma 5 to the infinite family $\left\{W_{n}^{\prime}: n \in \Sigma\right\}$ we get the existence of a set $A \subset \kappa$ such that $\mu(A) \geq \frac{1}{3}$ and $\left|\left\{n \in \Sigma: \alpha \in W_{n}^{\prime}\right\}\right|=\omega$ for every $\alpha \in A$. The set $A$ has the size $\kappa$ and therefore there exists a $\beta, \beta \in A$, such that $\gamma<\beta$. Consider the set $\Sigma^{\prime}=\left\{n \in \Sigma: \beta \in W_{n}^{\prime}\right\}$. The set $\Sigma^{\prime}$ is infinite and, by the definition of sets $W_{n}^{\prime}, s_{j} \cap T_{\beta} \neq \varnothing$ for every $j \in \Sigma^{\prime}$. Since the sets $s_{j}$ are pairwise disjoint, $\left|T_{\beta} \cap \bigcup\left\{s_{j}: j \in \Sigma^{\prime}\right\}\right|=\omega$. On the other hand, $s_{j} \cap T_{\gamma}=\varnothing$ for every $j \in \Sigma$. Since $\Sigma^{\prime} \subset \Sigma, T_{\gamma} \cap \bigcup\left\{s_{j}: j \in \Sigma^{\prime}\right\}=\varnothing$. Hence $\left|T_{\beta}-T_{\gamma}\right|=\omega$, which is impossible. The proof is complete.

COROLlARY 2. Suppose that there exists a $\kappa$-tower $\left\{T_{\alpha}: \alpha<\kappa\right\}$ such that for every $x \in[\omega]^{\omega}$ there exists an $\alpha, \alpha<\kappa$, such that $\left|x-T_{\alpha}\right|=\omega$. Then $\kappa$ is not a real-valued measurable cardinal.

2. Cofinality of ${ }^{\kappa} \lambda$. For arbitrary cardinals $\kappa, \lambda$ let ${ }^{\kappa} \lambda$ stand for the set of all functions from $\kappa$ into $\lambda$ endowed with the following order: $f \leq_{*} g$ iff $\mid\{\alpha<$ $\kappa: f(\alpha)>g(\alpha)\} \mid<\kappa$; we say that the function $g$ eventually dominates the function $f$. A set $\mathscr{C} \subset{ }^{\kappa} \lambda$ is cofinal in ${ }^{\kappa} \lambda$ if every $f \in{ }^{\kappa} \lambda$ is eventually dominated by some $g \in \mathscr{C}$. A set $\mathscr{U} \subset{ }^{\kappa} \lambda$ is unbounded in ${ }^{\kappa} \lambda$ if no $g \in{ }^{\kappa} \lambda$ eventually dominates all functions from $\mathscr{U}$. Let us put $\operatorname{cf}\left({ }^{\kappa} \lambda\right)$ to be the least cardinal number of a cofinal set in ${ }^{\kappa} \lambda$ and $\operatorname{ud}\left({ }^{\kappa} \lambda\right)$ to be the least cardinal number of an unbounded set in ${ }^{\kappa} \lambda$.

LEMMA 1. If $\delta=\operatorname{cf}(\lambda)$, then $\operatorname{cf}\left({ }^{\kappa} \lambda\right)=\operatorname{cf}\left({ }^{\kappa} \delta\right)$.

PROOF. Let $\left\{\xi_{\alpha}: \alpha<\delta\right\}$ be a strongly increasing sequence of ordinals converging to $\lambda$. Define two functions $G:{ }^{\kappa} \lambda \rightarrow{ }^{\kappa} \delta$ and $H:{ }^{\kappa} \lambda \rightarrow{ }^{\kappa} \delta$ by the rules

$$
G(f)(\beta)=\inf \left\{\alpha<\delta: f(\beta) \leq \xi_{\alpha}\right\} \quad \text { and } \quad H(g)(\beta)=\xi_{g(\beta)} .
$$

These two functions have the following properties:

(1) both are increasing, i.e., if $f_{1}, f_{2} \in{ }^{\kappa} \lambda$ and $f_{1} \leq_{*} f_{2}$, then $G\left(f_{1}\right) \leq_{*} G\left(f_{2}\right)$; if $g_{1}, g_{2} \in{ }^{\kappa} \delta$ and $g_{1} \leq_{*} g_{2}$, then $H\left(g_{1}\right) \leq_{*} H\left(g_{2}\right)$.

(2) if $f \in{ }^{\kappa} \lambda$, then $f \leq H(G(f))$; if $g \in{ }^{\kappa} \delta$, then $G(H(g))=g$.

The properties (1) and (2) imply that if $\mathscr{C} \subset{ }^{\kappa} \lambda$ is cofinal in ${ }^{\kappa} \lambda$, then $G(\mathscr{C})$ is cofinal in ${ }^{\kappa} \delta$ and if $\mathscr{C} \subset{ }^{\kappa} \delta$ is cofinal in ${ }^{\kappa} \delta$, then $H(\mathscr{C})$ is cofinal in ${ }^{\kappa} \lambda$.

The above lemma gives the right to assume that $\lambda$ is a regular cardinal when considering the cofinality of the set ${ }^{\kappa} \lambda$, which we shall do in the sequel without explicit mention. 
LEMMA 2. If $\kappa$ is a regular infinite cardinal, then $\operatorname{cf}\left({ }^{\kappa} \lambda\right)>\kappa$.

Proof. If $\lambda>\kappa$, then $\operatorname{cf}\left({ }^{\kappa} \lambda\right)=\lambda>\kappa$. If $\lambda=\kappa$, then a trivial diagonal argument shows that $\operatorname{cf}\left({ }^{\kappa} \kappa\right)>\kappa$. So let $\lambda<\kappa$. Let $\mathscr{F} \subset{ }^{\kappa} \lambda$ be of size $\leq \kappa$. Without loss of generality we may assume that for every $f \in \mathscr{F}$ and $\alpha \in \operatorname{rng} f$, $\left|f^{-1}(\{\alpha\})\right|=\kappa$. By a theorem of Sierpiński [S, p. 455] there are pairwise disjoint sets $A_{f, \alpha}$ such that $\left|A_{f, \alpha}\right|=\kappa$ and $A_{f, \alpha} \subset f^{-1}(\{\alpha\})$ for each $f \in \mathscr{F}$ and $\alpha \in \operatorname{rng} f$. Define $g \in{ }^{\kappa} \lambda$ by setting $g(\xi)=\alpha+1$ if $\xi \in A_{f, \alpha}$ for some $f \in \mathscr{F}$ and $\alpha \in \operatorname{rng} f$, and $g(\xi)=0$ otherwise. Clearly $g \Varangle_{*}$ for every $f \in \mathscr{F}$. Hence $\operatorname{cf}\left({ }^{\kappa} \lambda\right)>\kappa$.

LEMMA 3. If $\kappa, \rho$ are regular cardinals such that $\omega \leq \kappa \leq \rho$, then $\operatorname{cf}\left({ }^{\kappa} \lambda\right) \leq$ $\operatorname{cf}\left({ }^{\rho} \lambda\right)$.

ProOF. Let $\mathscr{C}$ be a cofinal set in ${ }^{\rho} \lambda$. Decompose $\rho$ into $\rho$ sets each of size $\kappa$, say $\rho=\bigcup\left\{R_{\alpha}: \alpha<\rho\right\}$, where $R_{\alpha} \cap R_{\beta}=\varnothing$ if $\alpha \neq \beta$ and $R_{\alpha}=\left\{\gamma_{\alpha \beta}: \beta<\kappa\right\}$. Every function $f \in{ }^{\rho} \lambda$ defines $\rho$ functions $f_{\alpha} \in{ }^{\kappa} \lambda, \alpha<\rho$, given by the rule: $f_{\alpha}(\beta)=f\left(\gamma_{\alpha \beta}\right)$. Take the family $\mathscr{F}=\left\{f_{\alpha}: f \in C\right.$ and $\left.\alpha<\rho\right\}$. This is a cofinal subset of ${ }^{\kappa} \lambda$. To see this, take arbitrary $g \in{ }^{\kappa} \lambda$. Define a function $h \in{ }^{\rho} \lambda$ by the rule $h(\xi)=g(\beta)$ whenever $\xi=\gamma_{\alpha \beta}$. Let $f, f \in \mathscr{C}$, be such that $h \leq_{*} f$. There exists an $\eta_{0}, \eta_{0}<\rho$, such that $h(\xi) \leq f(\xi)$ for every $\xi \geq \eta_{0}$. Hence there exists an $\alpha_{0}, \alpha_{0}<\rho$, such that $\gamma_{\alpha \beta}>\eta_{0}$ for every $\alpha \geq \alpha_{0}$ and $\beta<\kappa$. In consequence, $g(\beta)=h\left(\gamma_{\alpha \beta}\right) \leq f\left(\gamma_{\alpha \beta}\right)=f_{\alpha}(\beta)$ for every $\beta<\kappa$ and $\alpha \leq \alpha_{0}$. In particular, $g \leq_{*} f_{\alpha_{0}} \in \mathscr{F}$. The size of the family $\mathscr{F}$ is not greater than $\rho \cdot|\mathscr{C}|$. But because $|\mathscr{C}|>\rho,|\mathscr{F}| \leq|\mathscr{C}|$.

LEMMA 4. Let $\kappa, \lambda, \delta$ be regular infinite cardinals such that both $\lambda, \delta$ are less than $\kappa$. Suppose that there exists an unbounded sequence $\left\{g_{\alpha}: \alpha<\tau\right\}$ in ${ }^{\delta} \lambda$ such that $\alpha<\beta<\tau$ implies $g_{\alpha} \leq_{*} g_{\beta}$. Then $\operatorname{cf}\left({ }^{\kappa} \lambda\right) \geq \operatorname{cf}\left({ }^{\kappa} \tau\right)$.

PROOF. Decompose $\kappa$ into $\kappa$ pieces each of size $\delta$, say $\kappa=\bigcup\left\{R_{\alpha}: \alpha<\kappa\right\}$, where $R_{\alpha} \cap R_{\beta}=\varnothing$ if $\alpha \neq \beta$ and $R_{\alpha}=\left\{\gamma_{\alpha \beta}: \beta<\delta\right\}$. With every function $f \in{ }^{\kappa} \lambda$ we associate a function $h_{f} \in{ }^{\kappa} \tau$ in the following way:

Let $\alpha<\kappa$. Put $\phi_{\alpha}(\beta)=f\left(\gamma_{\alpha \beta}\right)$ for every $\beta<\delta$. Then $\phi_{\alpha} \in{ }^{\delta} \lambda$. Let $\beta_{0}=\inf \left\{\gamma: g_{\gamma} \Varangle_{*} \phi_{\alpha}\right\}$. Then we set $h_{f}(\alpha)=\beta_{0}$.

Take any set $\mathscr{F}$ cofinal in ${ }^{\kappa} \lambda$. We claim that the set $\left\{h_{f}: f \in \mathscr{F}\right\}$ is cofinal in ${ }^{\kappa} \tau$.

Let $s \in{ }^{\kappa} \tau$. Define a function $t \in{ }^{\kappa} \lambda$ by setting $t\left(\gamma_{\alpha \beta}\right)=g_{s(\alpha)}(\beta)$. Let $f \in \mathscr{F}$ be such that $t \leq_{*} f$. It is enough to show that $s \leq h_{f}$. Since $t \leq_{*} f$, there exists an $\alpha_{0}<\kappa$ such that $t\left(\gamma_{\alpha \beta}\right) \leq f\left(\gamma_{\alpha \beta}\right)$ for each $\beta<\delta$ and $\alpha, \alpha_{0} \leq \alpha<\kappa$. Take any $\alpha \geq \alpha_{0}$. Then $\phi_{\alpha}(\beta)=f\left(\gamma_{\alpha \beta}\right) \geq t\left(\gamma_{\alpha \beta}\right)=g_{s(\alpha)}(\beta)$ for each $\beta<\delta$. Hence $g_{s(\alpha)} \leq \phi_{\alpha}$ and therefore $\beta_{0}=\inf \left\{\gamma: g_{\gamma} \leq_{*} \phi_{\alpha}\right\} \geq s(\alpha)$ (because for every $\left.\gamma \leq s(\alpha), g_{\gamma} \leq_{*} g_{s(\alpha)} \leq \phi_{\alpha}\right)$. This means $s(\alpha) \leq h_{f}(\alpha)$ for every $\alpha, \alpha_{0} \leq \alpha<\kappa$.

To state our next lemma we will need some definitions concerning Boolean algebras.

A partition of a Boolean algebra $B$ is a maximal set of mutually incompatible nonzero elements of $B$.

A Boolean algebra $B$ is $(\kappa, \lambda)$-weakly distributive, if for every family $\left\{P_{\alpha}: \alpha<\kappa\right\}$ of partitions of $B$, each of size $\leq \lambda$, there is a partition $P$ of $B$ such that $\mid\{x \in$ $\left.P_{\alpha}: x \wedge p \neq 0\right\} \mid<\lambda$ for every $\alpha<\kappa$ and $p \in P$. 
LEMMA 5. Let I be a nontrivial $\nu$-complete ideal over a cardinal $\tau$ such that the quotient Boolean algebra $P(\tau) / I$ is not $(\kappa, \lambda)$-weakly distributive. Then $\operatorname{cf}\left({ }^{\kappa} \lambda\right) \geq \nu$.

PROOF. In virtue of Lemma 3 we may assume that the algebra $P(\tau) / I$ is $(\rho, \lambda)$ weakly distributive for every $\rho<\kappa$. Let $\left\{P_{\alpha}: \alpha<\kappa\right\}$ be a family of partitions of $P(\tau) / I$ each of size $\lambda$, such that there exists a nonzero element $p \in P(\tau) / I$ with the property that for every $g \in P(\tau) / I, 0<g \leq p$, there exists an $\alpha, \alpha<\kappa$ (in fact, $\kappa$ such $\alpha$ 's), such that $\left|\left\{x \in P_{\alpha}: x \wedge g \neq \varnothing\right\}\right|=\lambda$. Let us pass to $P(\tau)$ and choose a set $\hat{p} \in P(\tau)-I$ such that its equivalence class $[\hat{p}]$ is $p$. Similarly, for every $\alpha<\kappa$ and $x \in P_{\alpha}$ let us choose a set $\hat{x} \in P(\tau)-I$ such that $[\hat{x}]=x$ and put $\widehat{P}_{\alpha}=\left\{\hat{x}: x \in P_{\alpha}\right\}$. Finally, let us enumerate each $\widehat{P}_{\alpha}$, say $\widehat{P}_{\alpha}=\left\{\hat{x}_{\xi}: \xi<\lambda\right\}$. Our lemma becomes trivial when $\nu<\lambda$. So, assume $\lambda \leq \nu$. However this assumption allows to choose $\hat{x}$ 's in such a way that for every $\alpha<\kappa, \widehat{P}_{\alpha}$ consists of pairwise disjoint subsets of $\tau$ covering the whole $\tau$.

Let $\mathscr{F}$ be a cofinal subset of ${ }^{\kappa} \lambda$. For $f \in{ }^{\kappa} \lambda$ and $\alpha<\kappa$ put

$$
Z_{f, \alpha}=\bigcap\left\{\bigcup\left\{\hat{x}_{\xi}: \xi \leq f(\beta) \text { and } \hat{x}_{\xi} \in P_{\beta}\right\}: \alpha \leq \beta<\kappa\right\} .
$$

Note that $\hat{p} \cap Z_{f, \alpha} \in I$ for every $f \in{ }^{\kappa} \lambda$ and $\alpha<\kappa$. Since $\operatorname{cf}\left({ }^{\kappa} \lambda\right)>\kappa$, the family $\mathscr{L}=\left\{\hat{p} \cap Z_{f, \alpha}: f \in \mathscr{F}\right.$ and $\left.\alpha<\kappa\right\}$ has size $\leq|\mathscr{F}|$. This family covers $\hat{p}$. If $\gamma \in \hat{p}$, then let $g_{\gamma} \in{ }^{\kappa} \lambda$ be defined by the rule: $g_{\gamma}(\alpha)=\xi$ iff $\gamma \in \hat{x}_{\xi} \in P_{\alpha}$. Taking $f \in \mathscr{F}$ such that $g_{\gamma}(\alpha) \leq f(\alpha)$ for all $\alpha \geq \alpha_{0}$ we have $\gamma \in \hat{p} \cap Z_{f, \alpha_{0}}$. Since $\hat{p} \subset \bigcup \mathscr{L}$, $|\mathscr{L}| \geq \nu$. Hence $\nu \leq|\mathscr{L}| \leq|\mathscr{F}|$.

If $\mu$ is a $2^{\omega}$-additive measure on $P\left(2^{\omega}\right)$ and $I \subset P\left(2^{\omega}\right)$ is the ideal of null sets, then the Boolean algebra $P\left(2^{\omega}\right) / I$ is not $\left(\omega_{1}, \omega\right)$-weakly distributive. This, as a matter of fact, has been shown by T. Jech and K. Prikry [JP] (they proved that if $\left\{P_{\alpha}: \alpha<\omega_{1}\right\}$ are stochastically $\mu$-independent partitions of $P\left(2^{\omega}\right)$, then

$$
\mu\left(\bigcap\left\{\bigcup S_{\alpha}: \alpha<\omega_{1}\right\}\right)=0
$$

provided $S_{\alpha}$ is a finite subfamily of $P_{\alpha}$ for each $\alpha<\omega_{1}$; see Lemmas 2.6 and 2.7). Hence

THEOREM 1. Let $2^{\omega}$ be a real-valued measurable cardinal. If $\kappa, \lambda$ are cardinals such that $\omega \leq \kappa \leq 2^{\omega}, \kappa$ is regular and $\operatorname{cf}(\lambda)=\omega$, then $\operatorname{cf}\left({ }^{\kappa} \lambda\right)=2^{\omega}$.

In fact, from our lemmas one can get $\operatorname{cf}\left({ }^{\kappa} \lambda\right) \geq 2^{\omega}$. The equality is obtained by using additionally the following

THEOREM 2 (K. PRIKRY $[\mathbf{P}]$ ). If $2^{\omega}$ is a real-valued measurable cardinal, then $2^{\kappa}=2^{\omega}$ for every $\kappa<2^{\omega}$.

THEOREM 3. Let $2^{\omega}$ be a real-valued measurable cardinal. If $\kappa, \lambda$ are cardinals such that $\omega \leq \lambda \leq \kappa<2^{\omega}, \kappa$ is regular and $\operatorname{cf}(\lambda)>\omega$, then $\operatorname{cf}\left({ }^{\kappa} \lambda\right)<2^{\omega}$.

PROOF. In virtue of Lemma 1 we may assume that both cardinals $\kappa, \lambda$ are regular and $\omega<\lambda \leq \kappa<2^{\omega}$. Assume to the contrary that there is no cofinal set of size $<2^{\omega}$ in ${ }^{\kappa} \lambda$. Because $\lambda^{\kappa}=2^{\omega}$ one can enumerate ${ }^{\kappa} \lambda$ by $2^{\omega}$, say ${ }^{\kappa} \lambda=\left\{f_{\alpha}: \alpha<\right.$ $\left.2^{\omega}\right\}$. Now define inductively functions $g_{\alpha} \in{ }^{\kappa} \lambda, \alpha<2^{\omega}$, so that the following are satisfied:

(1) if $\alpha<\beta<2^{\omega}$, then $g_{\beta} \Varangle_{*} g_{\alpha}$, 
(2) $f_{\alpha}(\xi)<g_{\alpha}(\xi)$ for every $\xi<\kappa$ and $\alpha<2^{\omega}$.

Let $\mu$ be a $2^{\omega}$-additive probabilistic measure on $P\left(2^{\omega}\right)$. Because $\operatorname{cf}(\lambda)>\omega$, for every $\alpha<\kappa$ there exists a $g(\alpha)<\lambda$ such that the set $x_{\alpha}=\left\{\beta<2^{\omega}: g_{\beta}(\alpha) \leq g(\alpha)\right\}$ is of $\mu$-measure 1. Since $\kappa<2^{\omega}, x=\bigcap\left\{x_{\alpha}: \alpha<\kappa\right\}$ is of $\mu$-measure 1 , as well. There is an $\eta<2^{\omega}$ such that the function $f_{\eta}$ satisfies: $f_{\eta}(\alpha)=g(\alpha)$ for every $\alpha<\kappa$. Notice to the sequel that for every $\beta \in x$ and $\alpha<\kappa, g_{\beta}(\alpha) \leq f_{\eta}(\alpha)$. However, the set $x$ has size $2^{\omega}$, hence there exists a $\beta_{0} \in x$ such that $\eta<\beta_{0}$. In consequence, using (1) and (2), $g_{\beta_{0}} \leq f_{\eta}<g_{\eta}$ and $g_{\beta_{0}} \Varangle_{*} g_{\eta}$; a contradiction.

It remains to consider the cofinality of the set ${ }^{\omega} \omega$. It has been decided by $\mathrm{K}$. Kunen $[\mathbf{K}]$ : if $2^{\omega}$ is a real-valued measurable cardinal, then $\operatorname{cf}\left({ }^{\omega} \omega\right)<2^{\omega}$. However notice that the above proof works also for the set ${ }^{\omega} \omega$ (the set $x$ of the $\mu$-measure 1 can be obtained from Lemma $1, \S 1$ ). Because we do not need Prikry's theorem one can formulate a slight strengthening of Kunen's theorem:

THEOREM 4. If there exists a $\sigma$-additive probability measure on $P\left(2^{\omega}\right)$ such that each set of $\mu$-measure 1 has size $2^{\omega}$, then $\operatorname{cf}\left({ }^{\omega} \omega\right)<2^{\omega}$.

Finally, we have the following theorem.

THEOREM 5. Let $2^{\omega}$ be a real-valued measurable cardinal. Then for every cardinal $\delta, \omega \leq \delta<2^{\omega}$ there exist regular cardinals $\lambda_{1}, \lambda_{2}, \kappa$ such that $\delta<\lambda_{1}<\lambda_{2}<$ $\kappa<2^{\omega}$ and $\operatorname{cf}\left({ }^{\kappa} \lambda_{1}\right)=\operatorname{cf}\left({ }^{\kappa} \lambda_{2}\right)$.

PROOF. Define recursively $\eta_{n}, n<\omega$, by setting: $\eta_{0}=\mathrm{ud}\left({ }^{\delta^{+}} \delta^{+}\right)$and $\eta_{n+1}=$ $\operatorname{ud}\left({ }^{\eta_{n}} \eta_{n}\right)$. Then $\delta<\eta_{0}<\eta_{1}<\cdots<2^{\omega}$ because $\operatorname{ud}\left({ }^{\kappa} \lambda\right) \leq \operatorname{cf}\left({ }^{\kappa} \lambda\right)<2^{\omega}$ whenever $\operatorname{cf}(\lambda)>\omega$. Let $\kappa$ be a regular cardinal such that $\eta_{n}<\kappa<2^{\omega}$ for every $n<\omega$. In virtue of Lemma 4 we have $\operatorname{cf}\left({ }^{\kappa} \eta_{0}\right) \geq \operatorname{cf}\left({ }^{\kappa} \eta_{1}\right) \geq \cdots$. Because there does not exist a strongly decreasing sequence of ordinals one must have $\operatorname{cf}\left({ }^{\kappa} \eta_{i}\right)=\operatorname{cf}\left({ }^{\kappa} \eta_{i+1}\right)=$ ...

ACKNOWLEDGMEnT. The author is indebted to the referee for pointing out some errors in the original version.

\section{REFERENCES}

[vD] E. van Douwen, The integers and topology, Handbook of Set-Theoretic Topology (K. Kunen and J. Vaughan, eds.), North-Holland, Amsterdam, 1984, pp. 111-168.

[H] S. Hechler, Short complete nested sequences in $\beta N-N$ and small maximal almost disjoint families, General Topology Appl. 2 (1972), 139-149.

[J] T. Jech, Set theory, Academic Press, New York, 1978.

[JP] T. Jech and K. Prikry, Cofinality of the partial ordering of functions from $\omega_{1}$ into $\omega$ under eventual domination, Math. Proc. Cambridge Philos. Soc. 95 (1984), 25-33.

[KM] A. Kanamori and M. Magidor, The evolution of large cardinal axioms in set theory, Lecture Notes in Math., vol. 699, Springer-Verlag, Berlin and New York, pp. 99-275.

[K] K. Kunen, Inaccessibility properties of cardinals, doctoral dissertation, Stanford, 1968.

[P] K. Prikry, Ideals and powers of cardinals, Bull. Amer. Math. Soc. 81 (1975), 907-909.

[S] W. Sierpiński, Cardinal and ordinal numbers, Monogr. Mat., PWN, Warszawa, 1957.

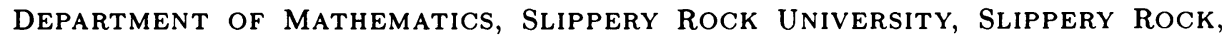
PENNSYLVANIA 16057

Department of Mathematics, Silesian University, Katowice, Poland 